\title{
Utility and safety of draining pleural effusions in mechanically ventilated patients: a systematic review and meta-analysis
}

\author{
Ewan C Goligher ${ }^{1,2}$, Jerome A Leis ${ }^{2}$, Robert A Fowler ${ }^{3}$, Ruxandra Pinto ${ }^{3}$, Neill KJ Adhikari ${ }^{3}$, Niall D Ferguson ${ }^{1,4^{*}}$
}

\begin{abstract}
Introduction: Pleural effusions are frequently drained in mechanically ventilated patients but the benefits and risks of this procedure are not well established.

Methods: We performed a literature search of multiple databases (MEDLINE, EMBASE, HEALTHSTAR, CINAHL) up to April 2010 to identify studies reporting clinical or physiological outcomes of mechanically ventilated critically ill patients who underwent drainage of pleural effusions. Studies were adjudicated for inclusion independently and in duplicate. Data on duration of ventilation and other clinical outcomes, oxygenation and lung mechanics, and adverse events were abstracted in duplicate independently.

Results: Nineteen observational studies $(N=1,124)$ met selection criteria. The mean $\mathrm{P}_{a} \mathrm{O}_{2}: \mathrm{F}_{i} \mathrm{O}_{2}$ ratio improved by 18\% (95\% confidence interval (Cl) 5\% to 33\%, $P^{2}=53.7 \%$, five studies including 118 patients) after effusion drainage. Reported complication rates were low for pneumothorax (20 events in 14 studies including 965 patients; pooled mean 3.4\%, 95\% Cl 1.7 to $6.5 \%, P^{2}=52.5 \%$ ) and hemothorax (4 events in 10 studies including 721 patients; pooled mean $1.6 \%, 95 \% \mathrm{Cl} 0.8$ to $3.3 \%, P^{2}=0 \%$ ). The use of ultrasound guidance (either real-time or for site marking) was not associated with a statistically significant reduction in the risk of pneumothorax (OR $=0.32 ; 95 \%$ $\mathrm{Cl} 0.08$ to 1.19). Studies did not report duration of ventilation, length of stay in the intensive care unit or hospital, or mortality.
\end{abstract}

Conclusions: Drainage of pleural effusions in mechanically ventilated patients appears to improve oxygenation and is safe. We found no data to either support or refute claims of beneficial effects on clinically important outcomes such as duration of ventilation or length of stay.

\section{Introduction}

Pleural effusions are common in the critically ill, occurring in over $60 \%$ of patients in some series [1,2]. Causes are multifactorial and include heart failure, pneumonia, hypoalbuminemia, intravenous fluid administration, atelectasis and positive pressure ventilation [1-5]. However, the impact of pleural effusions on the clinical outcomes of critically ill patients is unclear. Although the presence of pleural effusion on chest radiography has been associated with a longer duration of mechanical ventilation and ICU stay, the causal relationship is

\footnotetext{
* Correspondence: nferguson@mtsinai.on.ca

${ }^{1}$ Interdepartmental Division of Critical Care, Mount Sinai Hospital and the University Health Network, University of Toronto, 600 University Avenue, Toronto, Ontario, M5G 1X5, Canada

Full list of author information is available at the end of the article
}

unclear [2]. Data from animal studies suggest that pleural effusions reduce respiratory system compliance and increase intrapulmonary shunt with consequent hypoxemia [6-8]. In spontaneously breathing patients, drainage of large pleural effusions by thoracentesis generally produces only minor improvements in lung mechanics and oxygenation but significantly relieves dyspnea in most cases [9-17]. Complications of pleural drainage, such as pneumothorax, remain an important concern for many physicians, particularly in mechanically ventilated patients [18].

Given the uncertain benefits and risks of thoracentesis in mechanically ventilated patients, we conducted a systematic review of the literature to determine the impact of draining effusions in mechanically ventilated patients
C Biomed Central

(ㄷ) 2011 Goligher et al.; licensee BioMed Central Ltd. This is an open access article distributed under the terms of the Creative Commons Attribution License (http://creativecommons.org/licenses/by/2.0), which permits unrestricted use, distribution, and reproduction in any medium, provided the original work is properly cited. 
on clinical and physiologic outcomes and to ascertain the risk of serious procedural complications.

\section{Materials and methods \\ Data sources and searches}

We searched Medline (1954 to April 2010), EMBASE (1980 to April 2010), HealthStar (1966 to March 2010) and CINAHL (1990 to April 2010) using a sensitive search strategy combining $\mathrm{MeSH}$ headings and keywords to identify studies of critically ill, mechanically ventilated patients who underwent drainage of a pleural effusion (see Appendix). Search terms were defined a priori and by reviewing the $\mathrm{MeSH}$ terms of articles identified in preliminary literature searches. We contacted the authors of the papers identified and other opinion leaders to identify any other relevant studies. Two authors (ECG, JAL) independently reviewed the abstracts of all articles identified by the literature search and selected articles for detailed review of eligibility if either reviewer considered them potentially relevant. We also searched the bibliographies of all articles selected for detailed review and all relevant published reviews to find any other studies potentially eligible for inclusion.

\section{Study selection}

We selected observational studies or controlled trials meeting the following inclusion criteria: (1) adult patients receiving invasive mechanical ventilation; (2) pleural effusion confirmed by any imaging modality; (3) thoracentesis or placement of a catheter or tube to drain the pleural effusion; and, (4) clinical outcomes or physiological outcomes or complications reported. Clinical outcomes included duration of mechanical ventilation (primary outcome), mortality, ICU and hospital length of stay, and new clinical management actions based on pleural fluid analysis. Physiological outcomes included changes in oxygenation (ratio of partial pressure of oxygen in systemic arterial blood $\left(\mathrm{P}_{\mathrm{a}} \mathrm{O}_{2}\right)$ to inspired fraction of oxygen $\left(\mathrm{F}_{\mathrm{i}} \mathrm{O}_{2}\right)$, alveolar-arterial gradient of $\mathrm{P}_{\mathrm{a}} \mathrm{O}_{2}$, shunt fraction) and lung mechanics (peak inspiratory pressure, plateau pressure, tidal volume, respiratory rate, dynamic compliance). We recorded the occurrence of pneumothorax and hemothorax and other reported complications. We considered studies enrolling both mechanically ventilated and non-ventilated patients for inclusion if outcomes were reported separately for the mechanically ventilated subgroup. We excluded single case reports and studies of patients with pleural effusions that had absolute indications for drainage (for example, empyema, hemothorax, and so on). Each potential study was reviewed for eligibility in duplicate and independently by two authors (ECG, JAL); agreement between reviewers was assessed using Cohen's $\kappa$ [19]. Disagreements were resolved by consensus and consultation with a third author (NDF) when necessary.

\section{Data abstraction and quality assessment}

We collected data on patient demographics, admission diagnosis and severity of illness; study objective, setting, and design; ventilator settings; classification of pleural effusion (exudative vs. transudative); technique of drainage, including the use of imaging guidance, the level of training of the operator, and the type of drainage procedure performed; and outcomes. Only outcomes reported in mechanically ventilated patients were abstracted. For physiologic outcomes, we abstracted outcomes data and time of data collection before and after effusion drainage (see Additional file 1 for details [20]). One author (ECG) qualitatively assessed methodological quality based on the Newcastle-Ottawa Scale [21] and the guidelines developed by the MOOSE working group [22].

\section{Statistical analysis}

We aggregated outcomes data at the study level and performed statistical calculations with Review Manager (RevMan) 5.0 (2009; The Cochrane Collaboration, Oxford, UK) using random-effects models [23], which incorporate both within-study and between-study variation and generally provide more conservative effect estimates when heterogeneity is present. Data were pooled using the generic inverse variance method, which weights each study by the inverse of the variance of its effect estimate; the weight is adjusted in the presence of between-study heterogeneity. We verified analyses and constructed forest plots using the $\mathrm{R}$ statistical package, version 2.7.2 [24]. All statistical tests were two-sided. We considered $P<0.05$ as statistically significant in all analyses and report individual trial and summary results with $95 \%$ confidence intervals (CIs).

To conduct meta-analyses of risks of pneumothorax and hemothorax, we first converted the proportion of patients in each study with each complication to an odds. The standard error of each log odds, where odds $=\mathrm{X} /(\mathrm{n}$ $\mathrm{X})$ with $\mathrm{X}=$ events and $\mathrm{n}-\mathrm{X}=$ non-events, was calculated as $\sqrt{1 / X+1 /(n-X)}$. Natural log-transformed odds were pooled using the generic inverse variance method. For studies reporting zero events, we added 0.5 to both the numerator and denominator. Although values for this 'continuity correction' other than 0.5 may have superior statistical performance when comparing two treatment groups [25], previous work has shown that 0.5 gives the least biased estimator of the true log odds in a single treatment group situation [26]. The pooled log odds were converted back to a proportion. For the outcome of pneumothorax, we performed a sensitivity analysis restricting studies to those using simple thoracentesis 
(that is, no drain left in place). We conducted further sensitivity analyses using a Bayesian model with noninformative priors as implemented in Meta-Analyst software [27]. Each analysis used 500,000 iterations and converged. To compare complications for ultrasoundguided vs. physical landmark-guided effusion drainage, we calculated an odds ratio as exp (pooled log odds for ultrasound-guided group - pooled log odds for physical landmark-guided group) and compared the pooled log odds values using a z-test.

We report differences in $\mathrm{P}_{\mathrm{a}} \mathrm{O}_{2}: \mathrm{F}_{\mathrm{i}} \mathrm{O}_{2}$ ratio (P:F ratio) using the weighted mean of mean differences ( $P$ :F ratio after drainage - P:F ratio before drainage; a measure of absolute change) and the ratio of means (P:F ratio after drainage divided by P:F ratio before drainage; a measure of relative change) [28]. To estimate the standard errors of the mean differences as well as for the ratio of the means we assumed a correlation of 0.4 for the before and after measurements. Sensitivity analyses using alternate correlations of $0,0.3,0.5$ and 0.8 did not change the results qualitatively. We assessed between-study statistical heterogeneity for each outcome using the $I^{2}$ measure $[29,30]$ and considered statistical heterogeneity to be low for $I^{2}=25$ to $49 \%$, moderate for $I^{2}=50$ to $74 \%$, and high for $I^{2}>75 \%$ [30].

\section{Results}

Our search strategy identified 940 citations of interest, of which 58 reports were retrieved for full-text review (Figure 1). Nineteen studies met our selection criteria. There was excellent agreement between reviewers for study inclusion $(\kappa=0.88)$.

\section{Study characteristics}

The 19 included studies are summarized in Table 1; the authors of three studies provided additional information $[4,31,32]$. Four studies measured physiological effects of pleural drainage [31,33-35]; seven studies assessed the safety of thoracentesis [36-42]; and three studies assessed the accuracy of ultrasonographic prediction of pleural effusion size [43-45]. Four studies employed real-time ultrasound guidance [32-34,46] and eight studies employed ultrasound to mark the puncture site for thoracentesis [36,38-41,43,45,47]. Twelve studies used a one-time needle/catheter thoracentsis procedure, and six studies used a temporarily secured drainage catheter or thoracostomy tube.

The 19 included studies enrolled 1,690 patients, of which 1,124 patients received mechanical ventilation (median 40 mechanically ventilated patients per study, range 8 to 211). The mean age of enrolled patients ranged from 35 to 74 years. Of 494 patients in six studies reporting the type of effusion $[4,31,34,40,42,46], 42 \%$ were classified as exudative, $55 \%$ transudative (as defined in each study), and the remaining $3 \%$ had indeterminate biochemical findings.

\section{Methodological quality}

There were no randomized or non-randomized controlled trials of effusion drainage. Fifteen were prospective cohort studies [4,32-35,38-48] and four were retrospective cohort studies [31,36-38]. Most studies reported how patients were identified for inclusion and clearly outlined how the outcomes of pleural drainage were ascertained (see Additional file 1).

\section{Clinical outcomes}

Only data for mechanically ventilated patients were included. Given the absence of controlled studies, the effect of pleural drainage on duration of mechanical ventilation, ICU length of stay, or hospital length of stay could not be determined. One study $(n=44)$ compared ICU length of stay between patients with pleural effusion volume drainage greater vs. less than $500 \mathrm{~mL}$ and found no difference [44]. Fartoukh et al. reported that the results of thoracentesis $(n=113)$ changed the diagnosis in $43 \%$ of patients and modified the treatment plan in 31\% [4]. They found no significant reductions in duration of ICU stay or ICU mortality in patients whose management was altered by the results of thoracentesis compared to patients whose management was unchanged. Godwin et al. found that the results of thoracentesis affected management in 24 (75\%) of 32 cases [37].

\section{Oxygenation}

Six studies described the effects of thoracentesis on oxygenation (Table 2). One study of patients with severe acute respiratory distress syndrome included thoracentesis as part of a multimodal intervention for refractory hypoxemia that also mandated diuresis, optimization of conventional ventilation, permissive hypercapnia, and adjunctive measures such as prone positioning and inhaled nitric oxide. The effect of thoracentesis alone was unclear [48]. In the remaining five studies, the timing of gas exchange measurements, volume of drainage, ventilator settings, and the measured change in oxygenation after pleural drainage varied considerably. Metaanalysis (Figure 2) demonstrated an 18\% improvement in the P:F ratio after thoracentesis (95\% CI 5 to $33 \%$, $I^{2}=53.7 \%$, five studies including 118 patients) corresponding to an increase of $31 \mathrm{~mm} \mathrm{Hg}$ (95\% CI 6 to $55 \mathrm{~mm} \mathrm{Hg}, I^{2}=61.5 \%$, five studies including 118 patients).

Some studies identified possible predictors of improved oxygenation after thoracentesis. Roch et al. $(n=44)$ found that the increase in the P:F ratio correlated with the effusion volume drained $(\mathrm{r}=0.5, P=$ 0.01 ) in the subgroup of patients with pleural effusions 


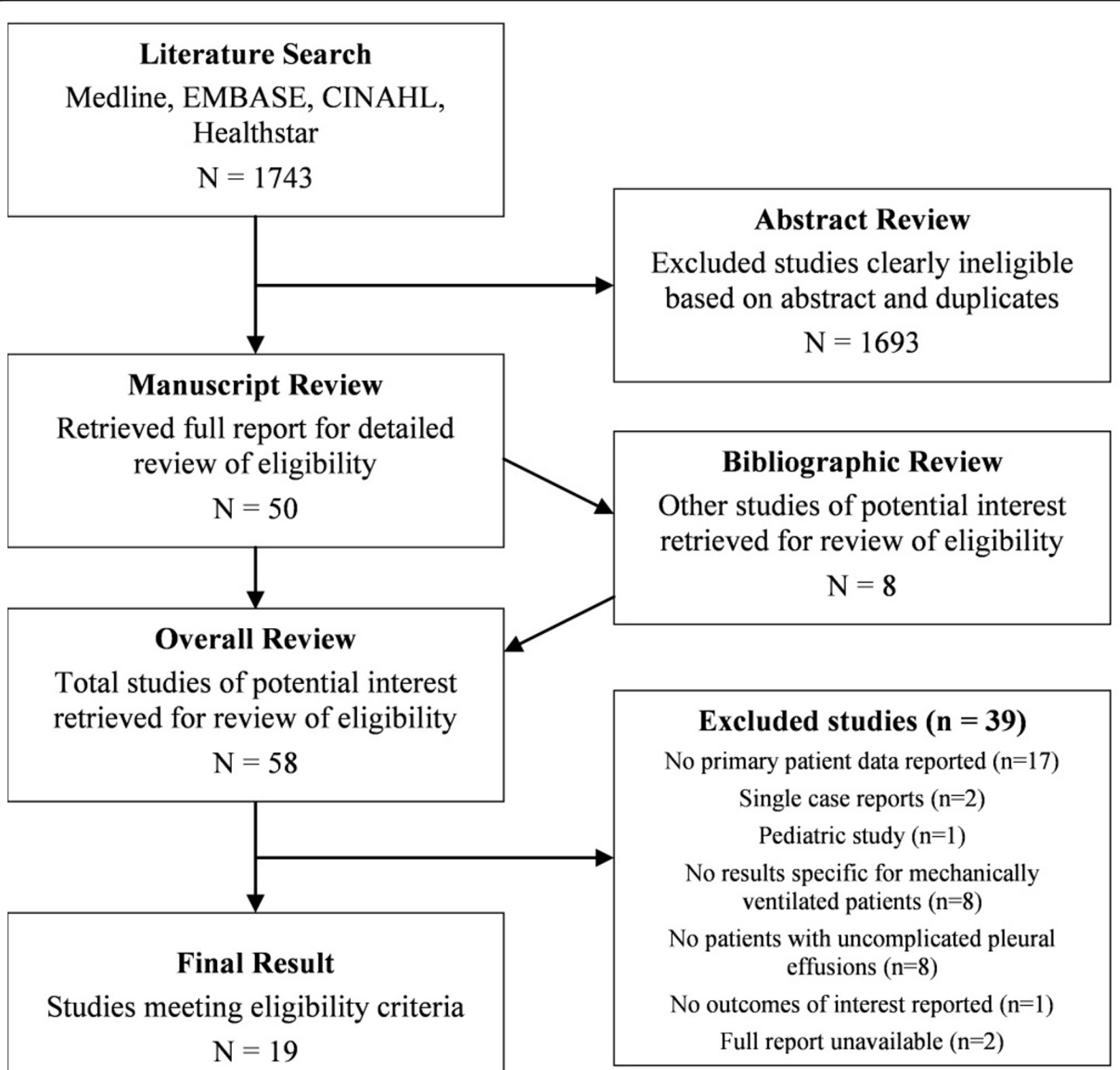

Figure 1 Summary of the study selection process

greater than $500 \mathrm{~mL}$ in size $(n=24)$. Conversely, Talmor et al. $(n=19)$ found no relationship between oxygenation response and the drained volume. In a multivariate analysis by De Waele et al. $(n=24)$, a P:F ratio less than $180 \mathrm{~mm} \mathrm{Hg}$ was the sole independent predictor of improved P:F ratio after thoracentesis [31].

\section{Lung mechanics}

Three studies reported on the association of thoracentesis with changes in lung mechanics (Table 3). Talmor et al. $(n=19)$ reported a $30 \%$ increase in dynamic compliance immediately after the procedure and Doelken et al. $(n=9)$ reported a trend toward increased dynamic compliance. Doelken et al. also found a statistically significant reduction in the work of inflation per cycle (calculated by integration of the pressure-time curve) after thoracentesis. Ahmed et al. $(n=22)$ observed a reduction in the respiratory rate after thoracentesis but there was no significant change in lung mechanics.

\section{Complications}

Sixteen studies reported complications associated with thoracentesis (Table 4), and all but one [33] prespecified detection of complications in the study protocol. 
Table 1 Summary of studies included in the systematic review

\begin{tabular}{|c|c|c|c|c|c|c|c|c|}
\hline Reference & Objective & Design & Population & $\mathrm{N}$ & $\begin{array}{l}\text { Mean } \\
\text { Age } \\
\text { (SD) }\end{array}$ & $\begin{array}{c}\text { Sex } N \\
(\% \\
\text { Female) }\end{array}$ & $\begin{array}{l}\text { Mechanical } \\
\text { Ventilation } \\
\mathrm{N}(\%)\end{array}$ & Intervention \\
\hline $\begin{array}{l}\text { Godwin } \\
1990[37]\end{array}$ & $\begin{array}{l}\text { Assess safety of } \\
\text { thoracentesis in } \\
\text { mechanically } \\
\text { ventilated patients }\end{array}$ & $\begin{array}{l}\text { Multi-centre } \\
\text { retrospective } \\
\text { cohort }\end{array}$ & $\begin{array}{l}\text { Mechanically ventilated } \\
\text { patients }\end{array}$ & 29 & $\begin{array}{c}\text { Range } 1 \\
\text { to } 88 \\
\text { years } \\
\text { (only } 1 \\
\text { patient } \\
\text { under } 25 \\
\text { years) }\end{array}$ & $\begin{array}{l}\text { Not } \\
\text { reported }\end{array}$ & $29(100 \%)$ & $\begin{array}{l}\text { Needle aspiration by } \\
\text { medical student or } \\
\text { resident }(84 \%) \text { or staff } \\
\text { intensivist }(16 \%) \text { without } \\
\text { imaging guidance }\end{array}$ \\
\hline Yu 1992 [47] & $\begin{array}{l}\text { Evaluate utility of } \\
\text { chest ultrasound in } \\
\text { diagnosis and } \\
\text { management of } \\
\text { critically ill patients }\end{array}$ & $\begin{array}{l}\text { Single-centre } \\
\text { prospective } \\
\text { cohort }\end{array}$ & $\begin{array}{l}\text { Critically ill patients (not } \\
\text { all admitted to } I C \cup^{a} \text { ) with } \\
\text { unclear findings on chest } \\
\text { radiography }\end{array}$ & 41 & $\begin{array}{l}56(18) \\
\text { years }\end{array}$ & $\begin{array}{c}10 \\
(24 \%)\end{array}$ & $14(34 \%)$ & $\begin{array}{l}\text { Needle aspiration after } \\
\text { puncture site marked } \\
\text { using ultrasound } \\
\text { guidance (performed in } \\
\text { patients with pleural } \\
\text { effusion on ultrasound) }\end{array}$ \\
\hline $\begin{array}{l}\text { McCartney } \\
1993[41]\end{array}$ & $\begin{array}{l}\text { Evaluate the safety of } \\
\text { thoracentesis in } \\
\text { mechanically } \\
\text { ventilated patients }\end{array}$ & $\begin{array}{l}\text { Single-centre } \\
\text { prospective } \\
\text { cohort }\end{array}$ & $\begin{array}{l}\text { Patients on mechanical } \\
\text { ventilation with a pleural } \\
\text { effusion and a clinical } \\
\text { indication for drainage }\end{array}$ & 26 & $\begin{array}{l}\text { Range } \\
19 \text { to } 92 \\
\text { years }\end{array}$ & $\begin{array}{l}\text { Not } \\
\text { reported }\end{array}$ & $26(100 \%)$ & $\begin{array}{l}\text { Needle aspiration by staff } \\
\text { intensivist; ultrasound } \\
\text { employed to mark } \\
\text { puncture site in some } \\
\text { cases (percentage } \\
\text { unknown) }\end{array}$ \\
\hline $\begin{array}{l}\text { Gervais } 1997 \\
\text { [36] }\end{array}$ & $\begin{array}{l}\text { Compare } \\
\text { pneumothorax rates } \\
\text { after thoracentesis } \\
\text { between ventilated } \\
\text { and spontaneously } \\
\text { breathing patients }\end{array}$ & $\begin{array}{l}\text { Single-centre } \\
\text { retrospective } \\
\text { cohort }\end{array}$ & $\begin{array}{l}\text { Patients who underwent } \\
\text { diagnostic thoracentesis } \\
\text { in the interventional } \\
\text { radiology suite over a } \\
\text { four-year period. Included } \\
\text { some pediatric patients. }\end{array}$ & 434 & $\begin{array}{l}\text { Range } 2 \\
\text { to } 90 \\
\text { years }\end{array}$ & $\begin{array}{c}184 \\
(42 \%)\end{array}$ & $90(21 \%)$ & $\begin{array}{l}\text { Needle aspiration by } \\
\text { resident or fellow under } \\
\text { staff supervision after } \\
\text { marking puncture site } \\
\text { using ultrasound } \\
\text { guidance }\end{array}$ \\
\hline $\begin{array}{l}\text { Guinard } \\
1997[48]\end{array}$ & $\begin{array}{l}\text { Evaluate the } \\
\text { prognostic utility of } \\
\text { the physiologic } \\
\text { response to a multiple } \\
\text { component } \\
\text { optimization strategy } \\
\text { in } \text { ARDS }^{b}\end{array}$ & $\begin{array}{l}\text { Single-centre } \\
\text { prospective } \\
\text { cohort }\end{array}$ & $\begin{array}{l}\text { Mechanically ventilated } \\
\text { patients with ARDS with } \\
\text { a lung injury score }>2.5 \\
\text { and severe hypoxemia } \\
\text { (mean SAPS } \|^{c} 46, \text { SD 14) }\end{array}$ & 36 & $\begin{array}{l}35(12) \\
\text { years }\end{array}$ & $\begin{array}{c}20 \\
(56 \%)\end{array}$ & $36(100 \%)$ & $\begin{array}{l}\text { Drainage of pleural } \\
\text { effusions where present } \\
\text { (exact method not } \\
\text { specified) along with } \\
\text { other maneuvers to } \\
\text { optimize gas exchange }\end{array}$ \\
\hline $\begin{array}{l}\text { Talmor } 1998 \\
\text { [35] }\end{array}$ & $\begin{array}{l}\text { Measure the effects of } \\
\text { pleural fluid drainage } \\
\text { on gas exchange and } \\
\text { pulmonary mechanics } \\
\text { in patients with severe } \\
\text { respiratory failure }\end{array}$ & $\begin{array}{l}\text { Single-centre } \\
\text { prospective } \\
\text { cohort }\end{array}$ & $\begin{array}{l}\text { Surgical ICU patients on } \\
\text { mechanical ventilation } \\
\text { with hypoxemia } \\
\text { unresponsive to } \\
\text { recruitment maneuver } \\
\left(\mathrm{PEEP}^{d} 20 \mathrm{~cm} \mathrm{H}_{2} \mathrm{O} \text { ) and }\right. \\
\text { pleural effusions on chest } \\
\text { radiograph (mean } \\
\text { APACHE } \|^{e} 21, \mathrm{SD} \text { 2) }\end{array}$ & 19 & $\begin{array}{l}68(4) \\
\text { years }\end{array}$ & $\begin{array}{l}\text { Not } \\
\text { reported }\end{array}$ & 19 (100\%) & $\begin{array}{l}\text { Large-bore tube } \\
\text { thoracostomy without } \\
\text { imaging guidance }\end{array}$ \\
\hline $\begin{array}{l}\text { Lichtenstein } \\
1999 \text { [39] }\end{array}$ & $\begin{array}{l}\text { Evaluate the safety of } \\
\text { ultrasound-guided } \\
\text { thoracentesis in } \\
\text { mechanically } \\
\text { ventilated patients }\end{array}$ & $\begin{array}{l}\text { Single-centre } \\
\text { prospective } \\
\text { cohort }\end{array}$ & $\begin{array}{l}\text { Medical ICU patients on } \\
\text { mechanical ventilation } \\
\text { with a pleural effusion } \\
\text { identified by routine } \\
\text { chest ultrasound and a } \\
\text { clinical indication for } \\
\text { drainage }\end{array}$ & 40 & $\begin{array}{l}64 \text { years } \\
\text { (SD not } \\
\text { reported) }\end{array}$ & $\begin{array}{c}22 \\
(55 \%)\end{array}$ & 40 (100\%) & $\begin{array}{l}\text { Needle aspiration by staff } \\
\text { intensivist marking } \\
\text { puncture site using } \\
\text { ultrasound guidance }\end{array}$ \\
\hline $\begin{array}{l}\text { Fartoukh } \\
2002[4]\end{array}$ & $\begin{array}{l}\text { Assess the impact of } \\
\text { routine thoracentesis } \\
\text { on diagnosis and } \\
\text { management }\end{array}$ & $\begin{array}{l}\text { Multi-centre } \\
\text { prospective } \\
\text { cohort }\end{array}$ & $\begin{array}{l}\text { Medical ICU patients } \\
\text { (median SAPS II 46, range } \\
30 \text { to } 56 \text { ) }\end{array}$ & 113 & $\begin{array}{c}59 \\
\text { (range } \\
42 \text { to } 68) \\
\text { years }\end{array}$ & $\begin{array}{c}54 \\
(48 \%)\end{array}$ & $68(60 \%)$ & $\begin{array}{l}\text { Needle aspiration without } \\
\text { imaging guidance }\end{array}$ \\
\hline $\begin{array}{l}\text { De Waele } \\
2003 \text { [31] }\end{array}$ & $\begin{array}{l}\text { Measure the effect of } \\
\text { drainage of pleural } \\
\text { effusions on } \\
\text { oxygenation }\end{array}$ & $\begin{array}{l}\text { Single-centre } \\
\text { retrospective } \\
\text { cohort }\end{array}$ & $\begin{array}{l}\text { Medical-surgical ICU } \\
\text { patients (mean APACHE ॥ } \\
21, S D \text { ) }\end{array}$ & 58 & $\begin{array}{l}53(19) \\
\text { years }\end{array}$ & $\begin{array}{c}19 \\
(33 \%)\end{array}$ & $24(41 \%)$ & $\begin{array}{l}\text { Small-bore pigtail } \\
\text { catheter insertion (61\%) } \\
\text { or tube thoracostomy } \\
\text { (39\%) by staff intensivist } \\
\text { without imaging- } \\
\text { guidance }\end{array}$ \\
\hline $\begin{array}{l}\text { Singh } 2003 \\
{[42]}\end{array}$ & $\begin{array}{l}\text { Evaluate the utility } \\
\text { and safety of a 16- } \\
\text { gauge catheter system } \\
\text { for draining pleural } \\
\text { effusions }\end{array}$ & $\begin{array}{l}\text { Multi-centre } \\
\text { prospective } \\
\text { cohort }\end{array}$ & $\begin{array}{l}\text { ICU patients with a large } \\
\text { pleural effusion thought } \\
\text { to contribute to } \\
\text { respiratory impairment }\end{array}$ & 10 & $\begin{array}{l}\text { Not } \\
\text { reported }\end{array}$ & $\begin{array}{l}\text { Not } \\
\text { reported }\end{array}$ & $8(80 \%)$ & $\begin{array}{l}\text { Small-bore catheter } \\
\text { insertion without } \\
\text { imaging guidance }\end{array}$ \\
\hline
\end{tabular}


Table 1 Summary of studies included in the systematic review (Continued)

\begin{tabular}{|c|c|c|c|c|c|c|c|c|}
\hline $\begin{array}{l}\text { Ahmed } \\
2004 \text { [33] }\end{array}$ & $\begin{array}{l}\text { Measure effects of } \\
\text { thoracentesis on } \\
\text { hemodynamic and } \\
\text { pulmonary physiology }\end{array}$ & $\begin{array}{l}\text { Single-centre } \\
\text { prospective } \\
\text { cohort }\end{array}$ & $\begin{array}{l}\text { Mechanically ventilated } \\
\text { surgical ICU patients with } \\
\text { a pulmonary artery } \\
\text { catheter and a large } \\
\text { pleural effusion and a } \\
\text { clinical indication for } \\
\text { drainage (mean APACHE } \\
\text { II } 17, \text { SD 6) }\end{array}$ & 22 & $\begin{array}{l}63(18) \\
\text { years }\end{array}$ & $\begin{array}{c}10 \\
(45 \%)\end{array}$ & $22(100 \%)$ & $\begin{array}{l}\text { Small-bore pigtail } \\
\text { catheter inserted under } \\
\text { real-time ultrasound } \\
\text { guidance }\end{array}$ \\
\hline $\begin{array}{l}\text { Mayo } 2004 \\
{[40]}\end{array}$ & $\begin{array}{l}\text { Evaluate the safety of } \\
\text { ultrasound-guided } \\
\text { thoracentesis in } \\
\text { mechanically } \\
\text { ventilated patients }\end{array}$ & $\begin{array}{l}\text { Single-centre } \\
\text { prospective } \\
\text { cohort }\end{array}$ & $\begin{array}{l}\text { Medical ICU patients on } \\
\text { mechanical ventilation } \\
\text { with a pleural effusion } \\
\text { and a clinical indication } \\
\text { for drainage }\end{array}$ & 211 & $\begin{array}{l}\text { Not } \\
\text { reported }\end{array}$ & $\begin{array}{l}\text { Not } \\
\text { reported }\end{array}$ & 211 (100\%) & $\begin{array}{l}\text { Needle aspiration, small- } \\
\text { bore pigtail catheter } \\
\text { insertion, or large-bore } \\
\text { tube thoracostomy by } \\
\text { medical housestaff under } \\
\text { staff supervision after } \\
\text { puncture site marked } \\
\text { using ultrasound } \\
\text { guidance }\end{array}$ \\
\hline Tu 2004 [46] & $\begin{array}{l}\text { Assess the need for } \\
\text { thoracentesis in febrile } \\
\text { medical ICU patients } \\
\text { and the utility of } \\
\text { ultrasonography for } \\
\text { diagnosing empyema }\end{array}$ & $\begin{array}{l}\text { Single-centre } \\
\text { prospective } \\
\text { cohort }\end{array}$ & $\begin{array}{l}\text { Medical ICU patients with } \\
\text { temperature }>38^{\circ} \mathrm{C} \text { for at } \\
\text { least eight hours and a } \\
\text { pleural effusion on chest } \\
\text { radiography and } \\
\text { ultrasound }\end{array}$ & 94 & $\begin{array}{c}66(19) \\
\text { years }\end{array}$ & $\begin{array}{c}39 \\
(41 \%)\end{array}$ & 81 (86\%) & $\begin{array}{l}\text { Needle aspiration under } \\
\text { real-time ultrasound } \\
\text { guidance }\end{array}$ \\
\hline $\begin{array}{l}\text { Roch } 2005 \\
{[44]}\end{array}$ & $\begin{array}{l}\text { Evaluate the accuracy } \\
\text { of ultrasonography to } \\
\text { predicting size of } \\
\text { pleural effusion }\end{array}$ & $\begin{array}{l}\text { Single-centre } \\
\text { prospective } \\
\text { cohort }\end{array}$ & $\begin{array}{l}\text { Medical-surgical ICU } \\
\text { patients on mechanical } \\
\text { ventilation with a clinical } \\
\text { indication for } \\
\text { thoracentesis }\end{array}$ & 44 & $60(11)$ & $\begin{array}{c}16 \\
(36 \%)\end{array}$ & 44 (100\%) & $\begin{array}{l}\text { Large-bore tube } \\
\text { thoracostomy without } \\
\text { imaging guidance }\end{array}$ \\
\hline $\begin{array}{l}\text { Vignon } 2005 \\
{[45]}\end{array}$ & $\begin{array}{l}\text { Evaluate the accuracy } \\
\text { of ultrasonography to } \\
\text { predicting size of } \\
\text { pleural effusion }\end{array}$ & $\begin{array}{l}\text { Single-centre } \\
\text { prospective } \\
\text { cohort }\end{array}$ & $\begin{array}{l}\text { Medical-surgical ICU } \\
\text { patients with suspected } \\
\text { pleural effusion based on } \\
\text { physical examination or } \\
\text { unexplained hypoxemia }\end{array}$ & 116 & $\begin{array}{l}60(20) \\
\text { years }\end{array}$ & $\begin{array}{c}41 \\
(35 \%)\end{array}$ & 68 (59\%) & $\begin{array}{l}\text { Needle aspiration after } \\
\text { puncture site marked } \\
\text { using ultrasound } \\
\text { guidance }\end{array}$ \\
\hline $\begin{array}{l}\text { Balik } 2006 \\
\text { [43] }\end{array}$ & $\begin{array}{l}\text { Assess the utility of } \\
\text { ultrasonography to } \\
\text { predict pleural } \\
\text { effusion size }\end{array}$ & $\begin{array}{l}\text { Single-centre } \\
\text { prospective } \\
\text { cohort }\end{array}$ & $\begin{array}{l}\text { Sedated and } \\
\text { mechanically ventilated } \\
\text { medical ICU patients with } \\
\text { a large pleural effusion } \\
\text { and a clinical indication } \\
\text { for thoracentesis (mean } \\
\text { APACHE II 20, SD 7) }\end{array}$ & 81 & $\begin{array}{l}60(15) \\
\text { years }\end{array}$ & $\begin{array}{c}34 \\
(42 \%)\end{array}$ & 81 (100\%) & $\begin{array}{l}\text { Needle aspiration (84\%) } \\
\text { or small-bore pigtail } \\
\text { catheter insertion (16\%) } \\
\text { by staff intensivist after } \\
\text { marking puncture site } \\
\text { using ultrasound } \\
\text { guidance }\end{array}$ \\
\hline $\begin{array}{l}\text { Doelken } \\
2006 \text { [34] }\end{array}$ & $\begin{array}{l}\text { Measure the effects of } \\
\text { thoracentesis on gas } \\
\text { exchange and } \\
\text { pulmonary mechanics }\end{array}$ & $\begin{array}{l}\text { Single-centre } \\
\text { prospective } \\
\text { cohort }\end{array}$ & $\begin{array}{l}\text { Mechanically ventilated } \\
\text { patients with a large } \\
\text { pleural effusion and a } \\
\text { clinical indication for } \\
\text { drainage }\end{array}$ & 8 & $\begin{array}{l}74(20) \\
\text { years }\end{array}$ & $5(63 \%)$ & $8(100 \%)$ & $\begin{array}{l}\text { Needle aspiration under } \\
\text { real-time ultrasound } \\
\text { guidance }\end{array}$ \\
\hline Tu 2006 [32] & $\begin{array}{l}\text { Describe the } \\
\text { epidemiology and } \\
\text { bacteriology of } \\
\text { parapneumonic } \\
\text { effusions and } \\
\text { empyema in the ICU }\end{array}$ & $\begin{array}{l}\text { Single-centre } \\
\text { prospective } \\
\text { cohort }\end{array}$ & $\begin{array}{l}\text { Medical ICU patients with } \\
\text { temperature }>38^{\circ} \mathrm{C} \text { for at } \\
\text { least eight hours and a } \\
\text { pleural effusion on chest } \\
\text { radiography and } \\
\text { ultrasound }\end{array}$ & 175 & $\begin{array}{l}65(18) \\
\text { years }\end{array}$ & $\begin{array}{c}65 \\
(37 \%)\end{array}$ & 148 (84\%) & $\begin{array}{l}\text { Needle aspiration under } \\
\text { real-time ultrasound } \\
\text { guidance }\end{array}$ \\
\hline $\begin{array}{l}\text { Liang } 2009 \\
\text { [38] }\end{array}$ & $\begin{array}{l}\text { Measure the } \\
\text { effectiveness and } \\
\text { safety of pigtail } \\
\text { catheters for drainage } \\
\text { of pleural effusions in } \\
\text { the ICU }\end{array}$ & $\begin{array}{l}\text { Single-centre } \\
\text { retrospective } \\
\text { cohort }\end{array}$ & $\begin{array}{l}\text { Medical-surgical ICU } \\
\text { patients with a pleural } \\
\text { effusion who underwent } \\
\text { pigtail catheter insertion } \\
\text { (mean APACHE II 17, } \\
\text { SD 7) }\end{array}$ & 133 & $\begin{array}{l}64(15) \\
\text { years }\end{array}$ & $\begin{array}{c}40 \\
(30 \%)\end{array}$ & 108 (81\%) & $\begin{array}{l}\text { Small-bore pigtail } \\
\text { catheter insertion by staff } \\
\text { intensivist after marking } \\
\text { puncture site using } \\
\text { ultrasound guidance }\end{array}$ \\
\hline
\end{tabular}

${ }^{a}$ ICU $=$ intensive care unit.

${ }^{b}$ ARDS $=$ acute respiratory distress syndrome.

${ }^{c}$ SAPS $=$ Simplified Acute Physiology Score.

${ }^{d}$ PEEP $=$ positive end-expiratory pressure.

${ }^{e} \mathrm{APACHE}=$ acute physiology and chronic health evaluation. 
Table 2 Summary of studies of oxygenation after thoracentesis in mechanically ventilated patients

\begin{tabular}{|c|c|c|c|c|c|c|c|c|}
\hline \multirow[t]{2}{*}{ Study } & \multirow{2}{*}{$\begin{array}{l}\mathrm{N} \text { on } \\
\mathrm{MV}^{a}\end{array}$} & \multirow{2}{*}{$\begin{array}{l}\text { PEEP }^{b} \\
\left(\mathrm{~cm} \mathrm{H}_{2} \mathrm{O}\right)\end{array}$} & \multirow{2}{*}{$\begin{array}{l}\text { Volume Drained } \\
\text { (mean } \pm \mathrm{SD})\end{array}$} & \multirow{2}{*}{$\begin{array}{l}\text { Time of Outcome } \\
\text { Measurement }\end{array}$} & \multirow[t]{2}{*}{ Variable } & \multicolumn{3}{|c|}{ Outcome $^{d}$} \\
\hline & & & & & & Before & After & $\begin{array}{c}P \text { - } \\
\text { value }\end{array}$ \\
\hline \multirow[t]{3}{*}{$\begin{array}{l}\text { Ahmed } \\
2004\end{array}$} & 22 & $\begin{array}{l}\text { Not } \\
\text { reported }\end{array}$ & $\begin{array}{l}1,262 \pm 762 \mathrm{~mL} \\
\text { (Initial drainage) }\end{array}$ & $\begin{array}{l}<1 \text { hour before and after } \\
\text { drainage }\end{array}$ & $\mathrm{P}_{\mathrm{a}} \mathrm{O}_{2}: \mathrm{F}_{\mathrm{i}} \mathrm{O}_{2}$ & $\begin{array}{l}245 \pm \\
103\end{array}$ & $270 \pm 101$ & $0.31^{c}$ \\
\hline & & & & & A-a Gradient & $\begin{array}{l}236 \pm \\
170\end{array}$ & $211 \pm 153$ & $0.52^{c}$ \\
\hline & & & & & Shunt Fraction & $\begin{array}{c}26.6 \pm \\
15.1\end{array}$ & $21.0 \pm 7.8$ & 0.03 \\
\hline $\begin{array}{l}\text { De } \\
\text { Waele } \\
2003 \\
\end{array}$ & 24 & $\begin{array}{l}\text { Not } \\
\text { reported }\end{array}$ & $\begin{array}{l}1,077 \mathrm{~mL} \text { (SD not reported) } \\
\text { (Over first } 24 \text { hours) }\end{array}$ & $\begin{array}{l}\text { Before and } 24 \text { hours after } \\
\text { drainage }\end{array}$ & $\mathrm{P}_{\mathrm{a}} \mathrm{O}_{2}: \mathrm{F}_{\mathrm{i}} \mathrm{O}_{2}$ & $\begin{array}{c}190 \pm \\
84\end{array}$ & $216 \pm 74$ & $0.16^{c}$ \\
\hline \multirow[t]{2}{*}{$\begin{array}{l}\text { Doelken } \\
2006\end{array}$} & 9 & 0 & $\begin{array}{l}1,575 \pm 450 \mathrm{~mL} \\
\text { (Initial drainage) }\end{array}$ & $\begin{array}{l}\text { Immediately before and after } \\
\text { procedure }\end{array}$ & $\mathrm{P}_{\mathrm{a}} \mathrm{O}_{2}: \mathrm{F}_{\mathrm{i}} \mathrm{O}_{2}{ }^{e}$ & $\begin{array}{l}96 \pm \\
29.7\end{array}$ & $102 \pm 21.9$ & 0.37 \\
\hline & & & & & A-a Gradient & $\begin{array}{c}226 \pm \\
99.6 \\
\end{array}$ & $217 \pm 85.2$ & 0.34 \\
\hline $\begin{array}{l}\text { Guinard } \\
1997 \\
\end{array}$ & 36 & $12 \pm 3$ & $\mathrm{n} / \mathrm{a}$ & $\begin{array}{l}6 \text { to } 12 \text { hours post- } \\
\text { optimization procedure }\end{array}$ & $\begin{array}{l}\text { Predefined gas } \\
\text { exchange response }{ }^{d}\end{array}$ & & $\begin{array}{l}53 \% \\
\text { responded }\end{array}$ & \\
\hline \multirow[t]{2}{*}{$\begin{array}{l}\text { Roch } \\
2005\end{array}$} & 44 & $6 \pm 2$ & $\begin{array}{l}730 \pm 440 \mathrm{~mL} \\
\text { (first three hours) }\end{array}$ & $\begin{array}{l}\text { Before and } 12 \text { hours after } \\
\text { drainage }\end{array}$ & $\begin{array}{l}\mathrm{P}_{\mathrm{a}} \mathrm{O}_{2}: \mathrm{F}_{\mathrm{i}} \mathrm{O}_{2} \text { (effusion } \\
<500 \mathrm{~mL})(\mathrm{N}=20)\end{array}$ & $\begin{array}{c}214 \pm \\
83\end{array}$ & $232 \pm 110$ & $0.47^{c}$ \\
\hline & & & & & $\begin{array}{l}\mathrm{P}_{\mathrm{a}} \mathrm{O}_{2}: \mathrm{F}_{\mathrm{i}} \mathrm{O}_{2} \text { (effusion } \\
>500 \mathrm{~mL})(\mathrm{N}=24)\end{array}$ & $\begin{array}{c}206 \pm \\
62 \\
\end{array}$ & $251 \pm 91$ & $<0.01$ \\
\hline $\begin{array}{l}\text { Talmor } \\
1998\end{array}$ & 19 & $17 \pm 1$ & $\begin{array}{l}863 \pm 164 \mathrm{~mL} \\
\text { (first eight hours) }\end{array}$ & $\begin{array}{l}\text { Immediately before and } 24 \\
\text { hours after drainage }\end{array}$ & $\mathrm{P}_{\mathrm{a}} \mathrm{O}_{2}: \mathrm{F}_{\mathrm{i}} \mathrm{O}_{2}$ & $\begin{array}{l}151.0 \pm \\
66.7\end{array}$ & $\begin{array}{c}244.5 \pm \\
126.8\end{array}$ & $<0.0001$ \\
\hline
\end{tabular}

${ }^{a} \mathrm{MV}$, mechanical ventilation.

${ }^{b}$ PEEP, positive end-expiratory pressure.

${ }^{c} P$-value not provided in the original paper; we calculated the $P$-value based on the data provided, assuming a correlation between the before and after measurements of 0.4 .

${ }^{d} \mathrm{P}_{\mathrm{a}} \mathrm{O}_{2}>100 \mathrm{~mm} \mathrm{Hg}$ on $\mathrm{F}_{\mathrm{i}} \mathrm{O}_{2} 1.0$ for at least six hours.

${ }^{a}$ Values are reported as mean \pm standard deviation.

e Original paper reported $\mathrm{P}_{\mathrm{a}} \mathrm{O}_{2}$ but all patients were on $\mathrm{F}_{\mathrm{i}} \mathrm{O}_{2}$ 1.0; we calculated $\mathrm{P}_{\mathrm{a}} \mathrm{O}_{2}: \mathrm{F}_{\mathrm{i}} \mathrm{O}_{2}$ from these data.

One study [32] included complication data from an earlier study that included some of the same patients [46]; the earlier study was removed from further analysis of complications. One study [4] did not report the number of procedures performed in mechanically ventilated patients and, therefore, could not be included in this calculation. The pooled risk of postthoracentesis pneumothorax was $3.4 \%$ (95\% CI 1.7 to 6.5\%; 20 events in 14 studies including 965 patients) (Figure 3). After excluding studies that employed a temporary drain to perform the drainage procedure, the pooled risk of pneumothorax was $4.3 \%$ (95\% CI 2.1 to $8.7 \%$; 12 events in 8 studies including 496 patients). The pooled risk of hemothorax was $1.6 \%$ (95\% CI 0.8 to $3.3 \%$; 4 events in 10 studies with 721 patients) (Figure 4). The use of ultrasound guidance was not associated with a reduction in pneumothorax (OR 0.32; 95\% CI 0.08 to 1.19). Sensitivity analyses using Bayesian models estimated an even lower risk of complications (pneumothorax: 1.3\%, 95\% credible interval $0.2 \%$ to $3.3 \%$; hemothorax $0.5 \%$, $95 \%$ credible interval $0 \%$ to $1.2 \%$ ).

\section{Discussion}

This systematic review demonstrates that pleural drainage in mechanically ventilated patients is associated with improved oxygenation and a reassuringly low risk of serious peri-procedural complications. There was some data to suggest that routine diagnostic thoracentesis may alter the diagnosis or management of this patient population. However, there were no data on the impact of pleural drainage on duration of mechanical ventilation, our primary outcome of interest. Furthermore, there were no controlled studies of thoracentesis for any clinical or physiological end-point. We conclude that there is no definite evidence to recommend for or against draining pleural effusions in mechanically ventilated patients to improve major clinical outcomes including mortality, duration of mechanical ventilation, or length of ICU or hospital stay.

Studies of the effect of effusion drainage on oxygenation report heterogeneous findings; these differences may be attributable to systematic variation in severity of pre-existing hypoxemia, lung and chest wall compliance, positive-end expiratory pressure settings, pleural effusion 


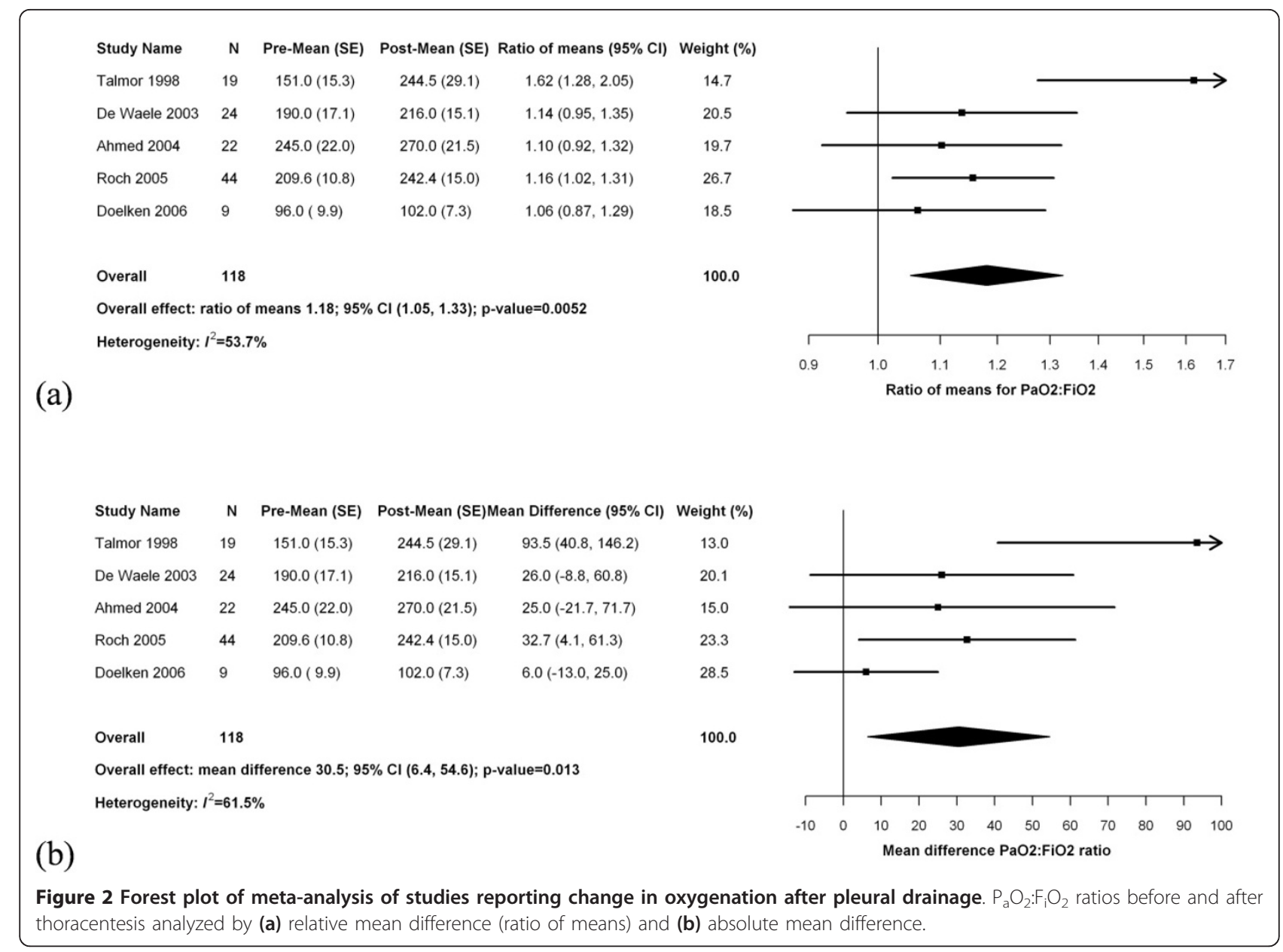

Table 3 Summary of studies of pulmonary mechanics after thoracentesis in mechanically ventilated patients

\begin{tabular}{|c|c|c|c|c|c|c|c|}
\hline \multirow[t]{2}{*}{ Study } & \multirow{2}{*}{$\begin{array}{c}\text { Proportion Mechanically } \\
\text { Ventilated }\end{array}$} & \multirow[t]{2}{*}{$\mathrm{N}$} & \multirow{2}{*}{$\begin{array}{l}\text { Time of Outcome } \\
\text { Measurement }\end{array}$} & \multirow[t]{2}{*}{ Variable } & \multicolumn{3}{|c|}{ Outcome $^{a}$} \\
\hline & & & & & Before & After & $\begin{array}{c}P- \\
\text { value }\end{array}$ \\
\hline \multirow[t]{2}{*}{$\begin{array}{l}\text { Ahmed } \\
2004\end{array}$} & $100 \%$ & 22 & $\begin{array}{l}<1 \text { hour before and after } \\
\text { thoracentesis }\end{array}$ & $\begin{array}{l}\text { Peak inspiratory pressure } \\
\left(\mathrm{cm} \mathrm{H}_{2} \mathrm{O}\right)\end{array}$ & $34.9 \pm 8.4$ & $35.9 \pm 12.5$ & $0.64^{b}$ \\
\hline & & & & Respiratory rate & $19.4 \pm 6.5$ & $15.5 \pm 6.3$ & 0.03 \\
\hline \multirow[t]{4}{*}{$\begin{array}{l}\text { Doelken } \\
2006\end{array}$} & $100 \%$ & 9 & $\begin{array}{l}\text { Immediately before and after } \\
\text { procedure }\end{array}$ & $\begin{array}{l}\text { Peak inspiratory pressure } \\
\left(\mathrm{cm} \mathrm{H}_{2} \mathrm{O}\right)\end{array}$ & $43.8 \pm 13.7$ & $40.8 \pm 10.6$ & 0.08 \\
\hline & & & & Plateau pressure $\left(\mathrm{cm} \mathrm{H}_{2} \mathrm{O}\right)$ & $20.0 \pm 9.0$ & $17.8 \pm 5.6$ & 0.19 \\
\hline & & & & $\begin{array}{l}\text { Dynamic compliance } \\
\left(\mathrm{L} / \mathrm{cm} \mathrm{H}_{2} \mathrm{O}\right)\end{array}$ & $14.5 \pm 5.3$ & $15.2 \pm 5.0$ & 0.12 \\
\hline & & & & $\begin{array}{l}\text { Ventilator work per cycle } \\
\text { (Joules) }\end{array}$ & $3.42 \pm 1.05$ & $2.99 \pm 0.81$ & 0.01 \\
\hline \multirow[t]{2}{*}{$\begin{array}{l}\text { Talmor } \\
1998\end{array}$} & $100 \%$ & 19 & $\begin{array}{l}\text { Immediately before and after } \\
\text { procedure }\end{array}$ & $\begin{array}{l}\text { Peak inspiratory pressure } \\
\left(\mathrm{cm} \mathrm{H}_{2} \mathrm{O}\right)\end{array}$ & $44.3 \pm 13.9$ & $42.9 \pm 18.7$ & $0.74^{b}$ \\
\hline & & & & $\begin{array}{l}\text { Dynamic compliance } \\
\left(\mathrm{L} / \mathrm{cm} \mathrm{H}_{2} \mathrm{O}\right)\end{array}$ & $27.1 \pm 15.3$ & $35.7 \pm 30.5$ & $<0.05$ \\
\hline
\end{tabular}

${ }^{a}$ Values are reported as mean \pm standard deviation.

${ }^{b} P$-value not provided in the original paper; we calculated the $P$-value based on the data provided, assuming a correlation between the before and after measurements of 0.4 . 
Table 4 Thoracentesis complication rates in mechanically ventilated patients

\begin{tabular}{|c|c|c|c|c|c|c|c|}
\hline Reference & $\begin{array}{l}\text { Operator } \\
\text { training }\end{array}$ & $\begin{array}{l}\text { Ultrasound } \\
\text { guidance }\end{array}$ & $\begin{array}{l}\text { Systematic } \\
\text { detection }^{a}\end{array}$ & $\begin{array}{c}\# \\
\text { Procedures } \\
\text { in MV } \\
\text { patients }\end{array}$ & $\begin{array}{l}\text { Pneumothorax } \\
\text { rate }\end{array}$ & $\begin{array}{l}\text { Hemothorax } \\
\text { rate }\end{array}$ & Additional findings \\
\hline $\begin{array}{l}\text { Godwin } \\
1990\end{array}$ & $\begin{array}{l}\text { Student or } \\
\text { resident } \\
\text { (84\%) or staff } \\
\text { intensivist } \\
(16 \%)\end{array}$ & None & Yes & 32 & $6.3 \%$ & $\mathrm{n} / \mathrm{a}^{b}$ & $\begin{array}{l}\text { The pneumothoraces occurred after } \\
\text { procedures performed by house staff No } \\
\text { tension pneumothoraces }\end{array}$ \\
\hline Yu 1992 & Not specified & $\begin{array}{l}\text { Puncture } \\
\text { site marked }\end{array}$ & Yes & 14 & $7.1 \%$ & $\mathrm{n} / \mathrm{a}$ & \\
\hline $\begin{array}{l}\text { McCartney } \\
1993\end{array}$ & $\begin{array}{l}\text { Staff } \\
\text { intensivist }\end{array}$ & $\begin{array}{l}\text { Puncture } \\
\text { site marked } \\
\text { in some } \\
\text { cases }\end{array}$ & Yes & 31 & $9.7 \%$ & $0 \%$ & No tension pneumothoraces \\
\hline Gervais 1997 & $\begin{array}{l}\text { Resident or } \\
\text { fellow }\end{array}$ & $\begin{array}{l}\text { Puncture } \\
\text { site marked }\end{array}$ & Yes & 90 & $6.7 \%$ & $\mathrm{n} / \mathrm{a}$ & $\begin{array}{l}\text { Only } 1 \% \text { of non-MV patients had } \\
\text { pneumothorax (difference in rates was } \\
\text { statistically significant) Only two of ten } \\
\text { pneumothoraces required chest tubes } \\
\text { (rest too small) }\end{array}$ \\
\hline $\begin{array}{l}\text { Lichtenstein } \\
1999\end{array}$ & $\begin{array}{l}\text { Staff } \\
\text { intensivist }\end{array}$ & $\begin{array}{l}\text { Puncture } \\
\text { site marked }\end{array}$ & Yes & 45 & $0 \%$ & $0 \%$ & \\
\hline $\begin{array}{l}\text { Fartoukh } \\
2002\end{array}$ & Not reported & None & Yes & Unknown & $\mathrm{n} / \mathrm{a}$ & $\mathrm{n} / \mathrm{a}$ & $\begin{array}{l}\text { Five of six reported pneumothoraces } \\
\text { occurred in patients on MV }\end{array}$ \\
\hline $\begin{array}{l}\text { De Waele } \\
2003\end{array}$ & $\begin{array}{l}\text { Staff } \\
\text { intensivist }\end{array}$ & None & Yes & 33 & $15 \%$ & $0 \%$ & $\begin{array}{l}\text { nine pneumothoraces in all patients } \\
\text { hemothorax }\end{array}$ \\
\hline Singh 2003 & Not specified & None & Yes & 12 & $0 \%$ & $0 \%$ & \\
\hline $\begin{array}{l}\text { Ahmed } \\
2004\end{array}$ & Not reported & $\begin{array}{l}\text { Real-time } \\
\text { guidance }\end{array}$ & No & 31 & $0.0 \%$ & $0 \%$ & \\
\hline Mayo 2004 & $\begin{array}{l}\text { Resident or } \\
\text { fellow }\end{array}$ & $\begin{array}{l}\text { Puncture } \\
\text { site marked }\end{array}$ & Yes & 232 & $1.3 \%$ & $0 \%$ & No tension pneumothoraces \\
\hline Tu 2004 & Not specified & $\begin{array}{l}\text { Real-time } \\
\text { guidance }\end{array}$ & Yes & Unknown & $0 \%$ & $\mathrm{n} / \mathrm{a}$ & $\begin{array}{l}\text { No pneumothoraces in all patients two } \\
\text { hemothoraces in all patients (Data } \\
\text { included in Tu 2006) }\end{array}$ \\
\hline Roch 2005 & Not specified & None & Yes & 44 & $0 \%$ & $4.5 \%$ & \\
\hline Vignon 2005 & Not specified & $\begin{array}{l}\text { Puncture } \\
\text { site marked }\end{array}$ & Yes & 17 & $0 \%$ & $0 \%$ & $\begin{array}{l}\text { Pneumothorax data available only on } 17 \\
\text { MV patients (unknown how many other } \\
\text { procedures were done on patients on } \\
\text { MV) }\end{array}$ \\
\hline Balik 2006 & $\begin{array}{l}\text { Staff } \\
\text { intensivist }\end{array}$ & $\begin{array}{l}\text { Puncture } \\
\text { site marked }\end{array}$ & Yes & 92 & $0.0 \%$ & $0 \%$ & \\
\hline Tu 2006 & Not specified & $\begin{array}{l}\text { Real-time } \\
\text { guidance }\end{array}$ & Yes & 184 & $0 \%$ & $1.1 \%$ & \\
\hline Liang 2009 & $\begin{array}{l}\text { Staff } \\
\text { intensivist }\end{array}$ & $\begin{array}{l}\text { Puncture } \\
\text { site marked }\end{array}$ & Yes & 108 & $0 \%$ & $\mathrm{n} / \mathrm{a}$ & $\begin{array}{l}\text { one hemothorax in all patients No } \\
\text { pneumothoraces in non-MV patients } \\
\text { three subcutaneous hematomas four } \\
\text { infections related to drainage seven } \\
\text { kinked catheters }\end{array}$ \\
\hline
\end{tabular}

${ }^{a}$ Protocol included pre-specified detection of complications of procedure including either chest radiography or chest ultrasound.

${ }^{b} \mathrm{n} / \mathrm{a}$, not available; that is, not reported in the paper or, if reported, the rate is not specific to patients on mechanical ventilation.

volume, and timing of observations. Studies of non-ventilated patients have documented relatively minor improvements in oxygenation after effusion drainage $[9,12,49,50]$. Small or moderate-sized effusions do not ordinarily cause significant hypoxemia because most $(75$ to $80 \%$ ) of the effusion volume is accommodated by the compliant chest wall and flattening of the diaphragm $[6,10,14,51]$. When chest wall compliance is reduced or the pleural effusion is large, effusions cause hypoxemia by collapsing adjacent lung with resultant physiologic shunt $[12,49]$. Drainage of pleural effusions may improve hypoxemia by allowing re-expansion of collapsed lung, which proceeds variably over the subsequent 24 hours [14] and may continue for several weeks [13,52]. In our review, one study [35] found significant improvement in oxygenation with thoracentesis by pre-selecting patients for study whose hypoxemia was refractory to high positive end-expiratory pressure (PEEP). This approach may 


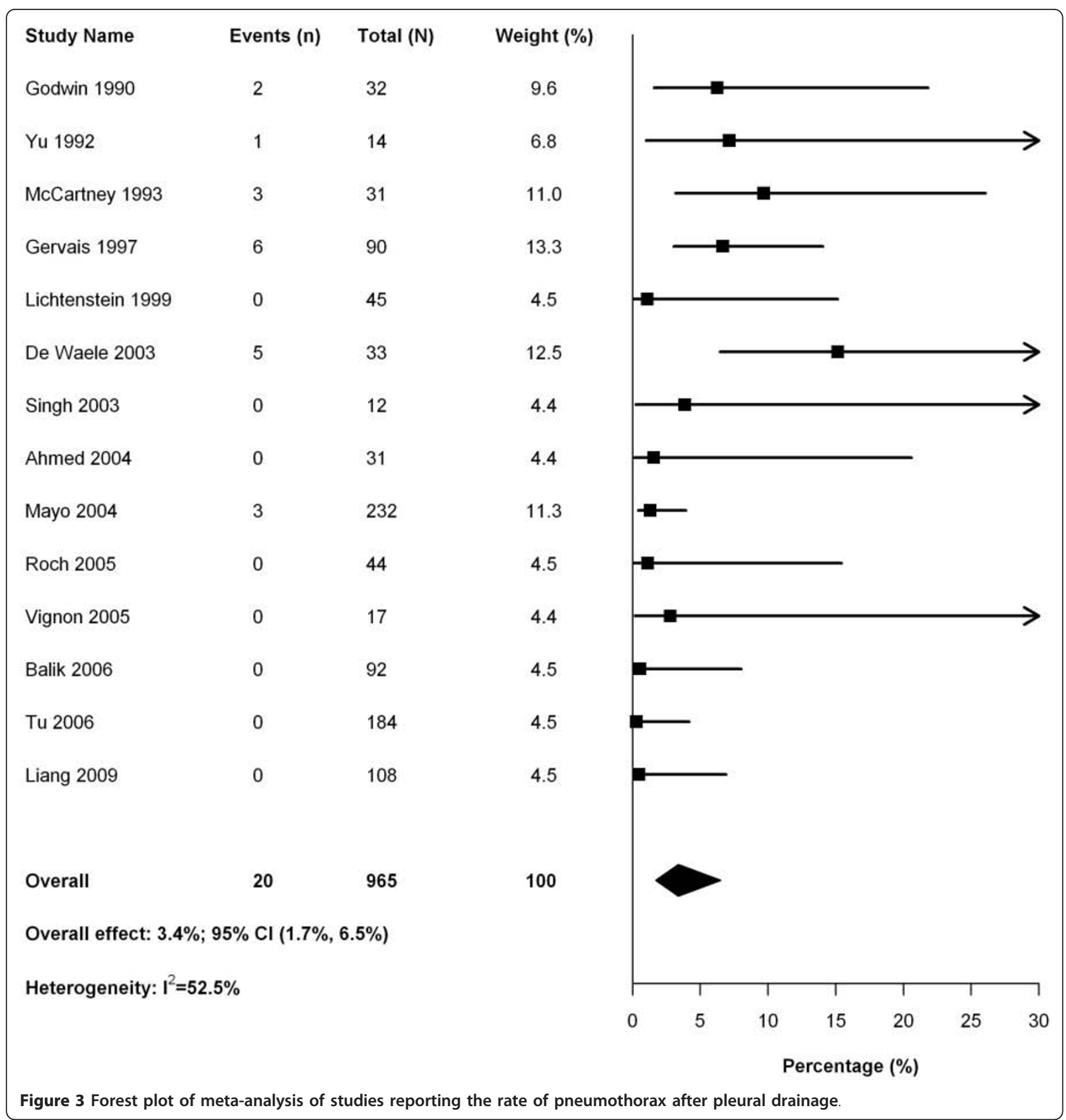

have identified patients with reduced chest wall or abdominal compliance whose oxygenation would be predicted to improve after effusion drainage [3]. In addition, the application of high PEEP may have promoted rapid recruitment of collapsed lung after effusion drainage.

A number of questions related to the impact of pleural effusion drainage on gas exchange remain unaddressed. These include the notion of the minimally important drainage volume and the use of maneuvers to re-expand previously collapsed lung after effusion drainage such as the application of PEEP. Also, it is unclear whether the degree of improvement in oxygenation after drainage depends on the severity of baseline hypoxemia or the total amount of fluid removed. In our systematic review, we did not perform meta-regression to assess the effect of either variable on improvement in oxygenation because of the limited number of studies, differences among studies in ventilator settings (making the interpretation of baseline hypoxemia difficult), and risk 


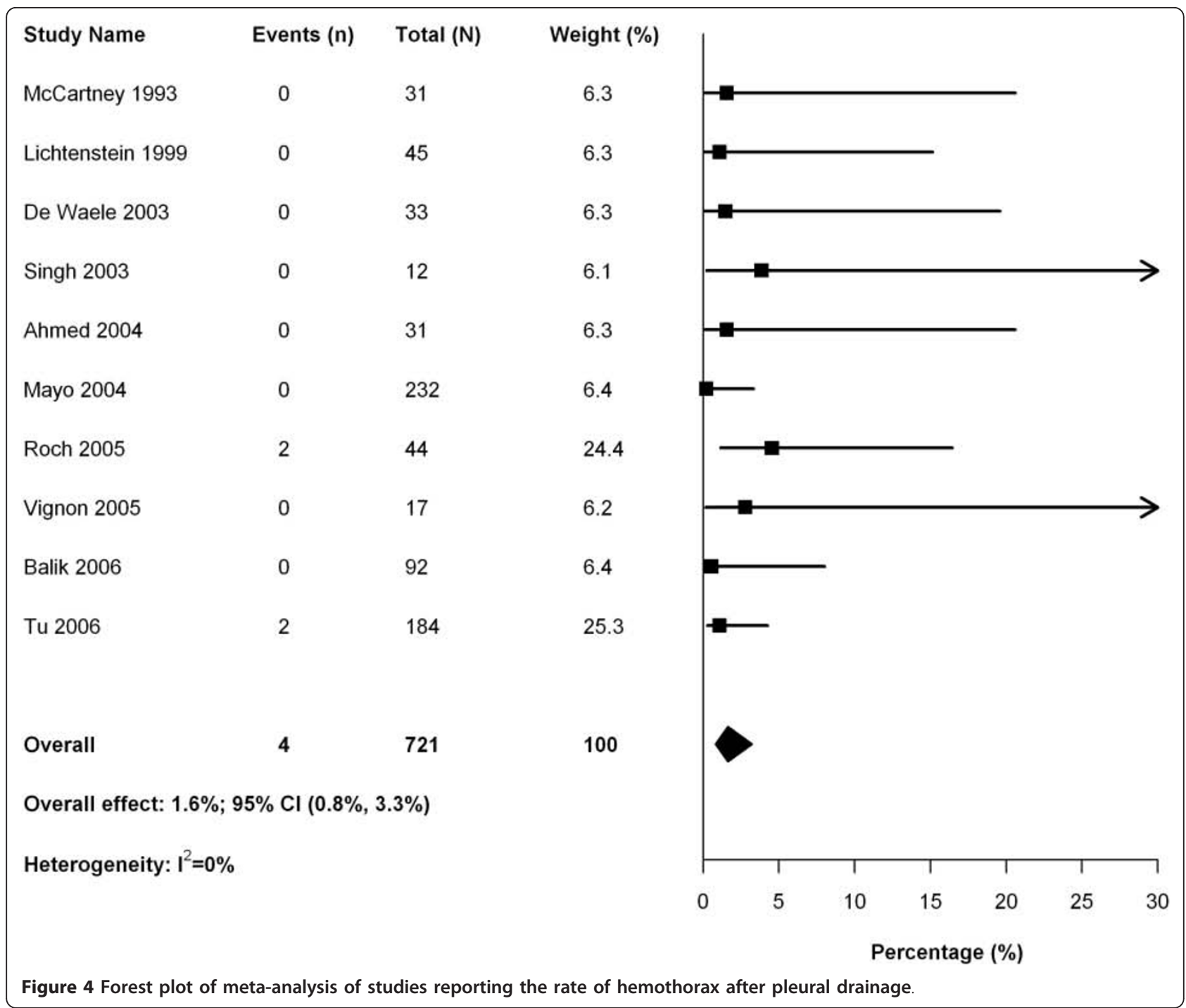

of ecological bias [53], since study-level meta-regression cannot determine whether patients within each study with more severe hypoxemia or more fluid drained benefited more.

Drainage of pleural effusions is sometimes proposed to accelerate weaning from mechanical ventilation. The underlying assumption is that pleural effusions decrease respiratory system compliance; drainage of effusions may therefore improve respiratory system mechanics and reduce ventilatory load. This review did not identify studies to strongly support or refute this hypothesis. Two uncontrolled studies [34,35] found relatively minor improvements in measures of compliance after effusion drainage, but it is unclear whether these changes would accelerate liberation from the ventilator. Data from spontaneously breathing patients show that drainage of effusions resulted in small improvements in lung volumes and static compliance [9-11,13-15,17], which would not likely explain the immediate relief of dyspnea reported by many patients.

Alternatively, draining pleural effusions may reduce the work of breathing by improving the mechanics of the diaphragm. Multiple authors have reported mechanical abnormalities of the diaphragm in the presence of pleural effusions including diaphragmatic inversion and paradoxical motion $[11,17,54-56]$. In a study of spontaneously breathing patients, Estenne et al. observed a marked increase in maximal inspiratory pressure immediately after thoracentesis [10] suggesting that diaphragmatic function might be impaired in the presence of a pleural effusion. They attributed the significant relief of dyspnea reported by patients after thoracentesis to improved diaphragm mechanics. In a recent study, the presence of paradoxical motion of the diaphragm in patients with pleural effusions predicted significantly greater improvements in dyspnea after thoracentesis 
[17]. Further research is necessary to replicate these findings in mechanically ventilated patients and to measure the potential benefit of pleural effusion drainage on duration of ventilation and other relevant clinical outcomes.

Clinicians may hesitate to perform thoracentesis in mechanically ventilated patients due to the risk of complications, particularly pneumothorax. This systematic review included studies that varied in setting, technique, use of ultrasound guidance and operator experience and found a low risk of complications in mechanically ventilated patients; the risk of pneumothorax was similar when studies were restricted to those performing simple thoracentesis with no drainage tube left in place. Sensitivity analysis using Bayesian methods found an even lower rate of complications than traditional metaanalysis. However, the true risk of complications may be higher outside of a study. We did not detect a reduction in complications associated with the use of periprocedural ultrasound guidance. A recent review of pneumothorax following thoracentesis among 24 studies of mostly spontaneously breathing patients found an overall rate of pneumothorax of $6.0 \%$ (95\% CI $4.6 \%$ to $7.8 \%$ ), of whom one-third required thoracostomy tube placement [57]. Ultrasound guidance was associated with a reduced risk of pneumothorax in that study. Although the risk of pneumothorax was non-significantly higher among patients receiving mechanical ventilation in that review, we found a lower rate among studies restricted to mechanically ventilated patients. This may be (1) because in our review drainage methods frequently included temporarily secured drainage catheters or thoracostomy tubes (that eliminate most pneumothoraces if they occur); (2) mechanically ventilated patients may be sedated for the procedure allowing for optimal positioning and reducing patient movement and therefore a lower risk of lung puncture; or (3) possibly due to other differences in operator characteristics or approach for patients receiving mechanical ventilation. Additionally, while we included all studies in the published metaanalysis that provided specific data on mechanically ventilated patients, we also incorporated data from several additional studies [4,31-33,38,41-47].

Strengths of this review include a broad literature search supplemented by contact with primary study investigators, consideration of a comprehensive set of outcomes, and consideration of alternate analytical approaches in sensitivity analyses. There are important limitations to this review related to the absence of controlled trials of pleural effusion drainage and lack of data on ventilator settings before and after drainage in some studies, which limited inferences regarding the effect of drainage on lung mechanics.

\section{Conclusions}

In summary, our systematic review did not identify any controlled studies of pleural effusion drainage in mechanically ventilated patients. Limited data suggest that pleural drainage is safe, may improve oxygenation, and under certain conditions may improve respiratory mechanics. We were unable to identify any evidence to support or refute the use of pleural drainage to promote liberation from mechanical ventilation. Further research is necessary and should focus on clarifying the physiological effects of pleural fluid drainage, the impact of the procedure on important clinical outcomes, the conditions under which a therapeutic response may be achieved, and the characteristics of those patients most likely to benefit from the procedure.

\section{Key messages}

- Pleural drainage is associated with minor improvements in oxygenation and lung mechanics.

- The complication rate from pleural drainage is very low. In our meta-analysis, the risk of postthoracentesis pneumothorax was 3.4\% (95\% CI 1.7 to $6.5 \%$; 20 events in 14 studies including 965 patients) and the pooled risk of hemothorax was $1.6 \%$ (95\% CI 0.8 to $3.3 \%$; 4 events in 10 studies including 721 patients).

- We could not find any studies reporting duration of ventilation or other clinically relevant ICU outcomes and further investigation is required to evaluate the benefit of pleural drainage in terms of liberation from mechanical ventilation.

\section{Additional material}

Additional file 1: Online appendix. Provides more in-depth details on literature search methods and results, data abstraction, quality assessment, and statistical analysis.

\section{Abbreviations}

APACHE: Acute Physiology And Chronic Health Evaluation; ARDS: Acute Respiratory Distress Syndrome; MV: mechanical ventilation; PEEP: Positive End-Expiratory Pressure; P:F ratio: $\mathrm{P}_{\mathrm{a}} \mathrm{O}_{2}: \mathrm{F}_{i} \mathrm{O}_{2}$ ratio; SAPS: Simplified Acute Physiology Score.

\section{Acknowledgements}

We would like to acknowledge Drs. E. Azoulay, J.J. De Waele, and C.Y. Tu for providing additional data for our systematic review.

Funding: Dr. Ferguson is supported by a New Investigator Award from the Canadian Institutes of Health Research (Ottawa, Canada). Dr. Fowler is supported by a Career Scientist Award from the Ontario Ministry of Health and Long-Term Care and a Phase II Clinician-Scientist Award from the Heart and Stroke Foundation of Canada.

\section{Author details}

${ }^{1}$ Interdepartmental Division of Critical Care, Mount Sinai Hospital and the University Health Network, University of Toronto, 600 University Avenue, Toronto, Ontario, M5G 1X5, Canada. ${ }^{2}$ Department of Medicine, Mount Sinai 
Hospital and the University Health Network, University of Toronto, 600 University Avenue, Toronto, Ontario, M5G 1X5, Canada. ${ }^{3}$ Department of Critical Care Medicine, Sunnybrook Health Sciences Centre, and the Interdepartmental Division of Critical Care, University of Toronto, 2075 Bayview Avenue, Toronto, Ontario, M4N 3M5, Canada. ${ }^{4}$ Department of Medicine, Division of Respirology, Mt. Sinai Hospital and the University Health Network, and the Interdepartmental Division of Critical Care, University of Toronto, 600 University Avenue, Toronto, Ontario, M5G 1X5, Canada.

\section{Authors' contributions}

EG participated in study design, data collection, data analysis and manuscript preparation. $J \mathrm{~L}$ participated in data collection and manuscript preparation. RF participated in study design, data analysis and manuscript preparation. RP participated in data analysis and manuscript preparation. NA participated in data analysis and manuscript preparation. NF participated in study design and manuscript preparation.

\section{Competing interests}

The authors declare that they have no competing interests.

Received: 9 November 2010 Revised: 12 January 2011

Accepted: 2 February 2011 Published: 2 February 2011

\section{References}

1. Azoulay E, Fartoukh M, Similowski T, Galliot R, Soufir L, Le Gall JR, Chevret $S$, Schlemmer B: Routine exploratory thoracentesis in ICU patients with pleural effusions: results of a French questionnaire study. J Crit Care 2001, 16:98-101.

2. Mattison LE, Coppage L, Alderman DF, Herlong JO, Sahn SA: Pleural effusions in the medical ICU: prevalence, causes, and clinical implications. Chest 1997, 111:1018-1023.

3. Graf J: Pleural effusion in the mechanically ventilated patient. Curr Opin Crit Care 2009, 15:10-17.

4. Fartoukh M, Azoulay E, Galliot R, Le Gall JR, Baud F, Chevret S, Schlemmer B: Clinically documented pleural effusions in medical ICU patients: how useful is routine thoracentesis? Chest 2002, 121:178-184

5. Soni N, Williams P: Positive pressure ventilation: what is the real cost? $\mathrm{Br}$ J Anaesth 2008, 101:446-457.

6. Krell WS, Rodarte JR: Effects of acute pleural effusion on respiratory system mechanics in dogs. J Appl Physiol 1985, 59:1458-1463.

7. Dechman G, Mishima M, Bates JH: Assessment of acute pleural effusion in dogs by computed tomography. J Appl Physiol 1994, 76:1993-1998.

8. Nishida O, Arellano R, Cheng DC, DeMajo W, Kavanagh BP: Gas exchange and hemodynamics in experimental pleural effusion. Crit Care Med 1999, 27:583-587.

9. Brown NE, Zamel N, Aberman A: Changes in pulmonary mechanics and gas exchange following thoracocentesis. Chest 1978, 74:540-542.

10. Estenne M, Yernault JC, De Troyer A: Mechanism of relief of dyspnea after thoracocentesis in patients with large pleural effusions. Am J Med 1983, 74:813-819.

11. Wang JS, Tseng CH: Changes in pulmonary mechanics and gas exchange after thoracentesis on patients with inversion of a hemidiaphragm secondary to large pleural effusion. Chest 1995, 107:1610-1614.

12. Perpina M, Benlloch E, Marco V, Abad F, Nauffal D: Effect of thoracentesis on pulmonary gas exchange. Thorax 1983, 38:747-750.

13. Altschule MD, Zamcheck $\mathrm{N}$ : The effects of pleural effusion on respiration and circulation in man. $J$ Clin Invest 1944, 23:325-331.

14. Light RW, Stansbury DW, Brown SE: The relationship between pleural pressures and changes in pulmonary function after therapeutic thoracentesis. Am Rev Respir Dis 1986, 133:658-661.

15. Gilmartin JJ, Wright AJ, Gibson GJ: Effects of pneumothorax or pleural effusion on pulmonary function. Thorax 1985, 40:60-65.

16. Light RW, Stansbury DW, Brown SE: Changes in pulmonary function following therapeutic thoracocentesis. Chest 1981, 80:375.

17. Wang LM, Cherng JM, Wang JS: Improved lung function after thoracocentesis in patients with paradoxical movement of a hemidiaphragm secondary to a large pleural effusion. Respirology 2007, 12:719-723.
18. Peek GJ, Firmin RK: Reducing morbidity from insertion of chest drains. Patients must be disconnected from positive airways pressure before insertion of drains. BMJ 1997, 315:313.

19. Cohen J: Weighted kappa: nominal scale agreement with provision for scaled disagreement or partial credit. Psychol Bull 1968, 70:213-220.

20. Wallace BC, Schmid CH, Lau J, Trikalinos TA: Meta-Analyst: software for meta-analysis of binary, continuous and diagnostic data. BMC Med Res Methodol 2009, 9:80.

21. The Newcastle-Ottawa Scale (NOS) for assessing the quality of nonrandomised studies in meta-analyses. [http://www.ohri.ca/programs/ clinical_epidemiology/oxford.htm].

22. Stroup DF, Berlin JA, Morton SC, Olkin I, Williamson GD, Rennie D, Moher D, Becker BJ, Sipe TA, Thacker SB: Meta-analysis of observational studies in epidemiology: a proposal for reporting. Meta-analysis Of Observational Studies in Epidemiology (MOOSE) group. JAMA 2000, 283:2008-2012.

23. DerSimonian R, Laird N: Meta-analysis in clinical trials. Control Clin Trials 1986, 7:177-188.

24. The R Project for Statistical Computing. [http://www.r-project.org].

25. Sweeting MJ, Sutton AJ, Lambert PC: What to add to nothing? Use and avoidance of continuity corrections in meta-analysis of sparse data. Stat Med 2004, 23:1351-1375.

26. Cox DR: The Analysis of Binary Data London: Methuen \& Co. Ltd; 1970.

27. Wallace BC, Schmid CH, Lau J, Trikalinos TA: Meta-Analyst: software for meta-analysis of binary, continuous and diagnostic data. BMC Med Res Methodol 2009, 9:80.

28. Friedrich JO, Adhikari NK, Beyene J: The ratio of means method as an alternative to mean differences for analyzing continuous outcome variables in meta-analysis: a simulation study. BMC Med Res Methodol 2008, 8:32

29. Higgins JP, Thompson SG: Quantifying heterogeneity in a meta-analysis. Stat Med 2002, 21:1539-1558.

30. Higgins JP, Thompson SG, Deeks JJ, Altman DG: Measuring inconsistency in meta-analyses. BMJ 2003, 327:557-560.

31. De Waele JJ, Hoste E, Benoit D, Vandewoude K, Delaere S, Berrevoet F, Colardyn F: The effect of tube thoracostomy on oxygenation in ICU patients. J Intensive Care Med 2003, 18:100-104.

32. Tu CY, Hsu WH, Hsia TC, Chen HJ, Chiu KL, Hang LW, Shih CM: The changing pathogens of complicated parapneumonic effusions or empyemas in a medical intensive care unit. Intensive Care Med 2006, 32:570-576.

33. Ahmed SH, Ouzounian SP, Dirusso S, Sullivan T, Savino J, Del Guercio L: Hemodynamic and pulmonary changes after drainage of significant pleural effusions in critically ill, mechanically ventilated surgical patients. J Trauma 2004, 57:1184-1188.

34. Doelken P, Abreu R, Sahn SA, Mayo PH: Effect of thoracentesis on respiratory mechanics and gas exchange in the patient receiving mechanical ventilation. Chest 2006, 130:1354-1361.

35. Talmor M, Hydo L, Gershenwald JG, Barie PS: Beneficial effects of chest tube drainage of pleural effusion in acute respiratory failure refractory to positive end-expiratory pressure ventilation. Surgery 1998, 123:137-143.

36. Gervais DA, Petersein A, Lee MJ, Hahn PF, Saini S, Mueller PR: US-guided thoracentesis: requirement for postprocedure chest radiography in patients who receive mechanical ventilation versus patients who breathe spontaneously. Radiology 1997, 204:503-506.

37. Godwin JE, Sahn SA: Thoracentesis: a safe procedure in mechanically ventilated patients. Ann Intern Med 1990, 113:800-802.

38. Liang SJ, Tu CY, Chen HJ, Chen CH, Chen W, Shih CM, Hsu WH: Application of ultrasound-guided pigtail catheter for drainage of pleural effusions in the ICU. Intensive Care Med 2009, 35:350-354.

39. Lichtenstein D, Hulot JS, Rabiller A, Tostivint I, Meziere G: Feasibility and safety of ultrasound-aided thoracentesis in mechanically ventilated patients. Intensive Care Med 1999, 25:955-958.

40. Mayo PH, Goltz HR, Tafreshi M, Doelken P: Safety of ultrasound-guided thoracentesis in patients receiving mechanical ventilation. Chest 2004, 125:1059-1062.

41. McCartney JP, Adams JW, Hazard PB: Safety of thoracentesis in mechanically ventilated patients. Chest 1993, 103:1920-1921.

42. Singh K, Loo S, Bellomo R: Pleural drainage using central venous catheters. Crit Care 2003, 7:R191-4. 
43. Balik $M$, Plasil P, Waldauf $P$, Pazout J, Fric M, Otahal M, Pachl J: Ultrasound estimation of volume of pleural fluid in mechanically ventilated patients. Intensive Care Med 2006, 32:318-321.

44. Roch A, Bojan M, Michelet P, Romain F, Bregeon F, Papazian L, Auffray JP: Usefulness of ultrasonography in predicting pleural effusions $>500 \mathrm{~mL}$ in patients receiving mechanical ventilation. Chest 2005, 127:224-232.

45. Vignon P, Chastagner C, Berkane V, Chardac E, Francois B, Normand S, Bonnivard M, Clavel M, Pichon N, Preux PM, Maubon A, Gastinne H: Quantitative assessment of pleural effusion in critically ill patients by means of ultrasonography. Crit Care Med 2005, 33:1757-1763.

46. Tu CY, Hsu WH, Hsia TC, Chen HJ, Tsai KD, Hung CW, Shih CM: Pleural effusions in febrile medical ICU patients: chest ultrasound study. Chest 2004, 126:1274-1280.

47. Yu CJ, Yang PC, Chang DB, Luh KT: Diagnostic and therapeutic use of chest sonography: value in critically ill patients. AJR Am J Roentgenol 1992, 159:695-701.

48. Guinard N, Beloucif S, Gatecel C, Mateo J, Payen D: Interest of a therapeutic optimization strategy in severe ARDS. Chest 1997, 111:1000-1007.

49. Agusti AG, Cardus J, Roca J, Grau JM, Xaubet A, Rodriguez-Roisin R: Ventilation-perfusion mismatch in patients with pleural effusion: effects of thoracentesis. Am J Respir Crit Care Med 1997, 156:1205-1209.

50. Karetzky MS, Kothari GA, Fourre JA, Khan AU: Effect of thoracentesis on arterial oxygen tension. Respiration 1978, 36:96-103.

51. Anthonisen NR, Martin RR: Regional lung function in pleural effusion. Am Rev Respir Dis 1977, 116:201-207.

52. Yoo OH, Ting EY: The Effects of Pleural Effusion on Pulmonary Function. Am Rev Respir Dis 1964, 89:55-63.

53. Greenland S, Morgenstern H: Ecological bias, confounding, and effect modification. Int J Epidemiol 1989, 18:269-274.

54. Mulvey RB: The Effect of Pleural Fluid on the Diaphragm. Radiology 1965, 84:1080-1086

55. Cooper JC, Elliott ST: Pleural effusions, diaphragm inversion, and paradox: new observations using sonography. AJR Am J Roentgenol 1995, 164:510.

56. Altschule MD: Some neglected aspects of respiratory function in pleural effusions. The diaphragmatic arch. Chest 1986, 89:602.

57. Gordon CE, Feller-Kopman D, Balk EM, Smetana GW: Pneumothorax following thoracentesis: a systematic review and meta-analysis. Arch Intern Med 2010, 170:332-339.

doi:10.1186/cc10009

Cite this article as: Goligher et al: Utility and safety of draining pleural effusions in mechanically ventilated patients: a systematic review and meta-analysis. Critical Care 2011 15:R46.

\section{Submit your next manuscript to BioMed Central and take full advantage of:}

- Convenient online submission

- Thorough peer review

- No space constraints or color figure charges

- Immediate publication on acceptance

- Inclusion in PubMed, CAS, Scopus and Google Scholar

- Research which is freely available for redistribution 